\title{
Early esophagogastroduodenoscopy is associated with better Outcomes in upper gastrointestinal bleeding: a nationwide study
}

\section{(ㄷ)(구)(우}

\section{Authors}

Sushil K. Garg ${ }^{1}$, Chimaobi Anugwom¹, James Campbell' ${ }^{1}$, Vaibhav Wadhwa², Nancy Gupta ${ }^{3}$, Rocio Lopez ${ }^{4}$, Sukhman Shergill ${ }^{5}$, Madhusudhan R. Sanaka6

\section{Institutions}

1 Department of Internal Medicine, University of Minnesota Twin Cities, Minneapolis, Minnesota, United States

2 Department of Internal Medicine, Fairview Hospital, Cleveland Clinic, Cleveland, Ohio, United States

3 Department of Gastroenterology, University of Iowa, lowa City, lowa, United States

4 Quantitative Health Sciences, Cleveland Clinic, Cleveland, Ohio, United States

5 All India Institute of Medical Sciences, Medicine, New Delhi, India

6 Digestive Disease Institute, Department of Gastroenterology \& Hepatology, The Cleveland Clinic, Cleveland, Ohio, United States

submitted 8.7.2016

accepted after revision $\quad 2.11 .2016$

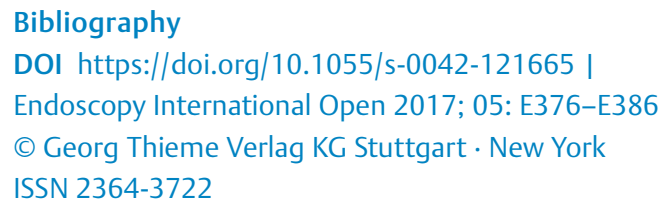

Corresponding author

Madhu Sanaka, MD, FACG, FASGE, Director of Endoscopy

Research, Department of Gastroenterology, Desk Q3, 9500

Euclid Avenue, Cleveland, $\mathrm{OH} 44195$

Fax: +1-216-444-6283

sanakam@ccf.org

\section{ABSTRACT}

Background and study aims We analyzed NIS (National Inpatient Sample) database from 2007-2013 to determine if early esophagogastroduodenoscopy (EGD) (24 hours) for upper gastrointestinal bleeding improved the outcomes in terms of mortality, length of stay and costs.

Patients and methods Patients were classified as having upper gastrointestinal hemorrhage by querying all diagnostic codes for the ICD-9-CM codes corresponding to upper gastrointestinal bleeding. For these patients, performance of EGD during admission was determined by querying all procedural codes for the ICD-9-CM codes corresponding to EGD; early EGD was defined as having EGD performed within 24 hours of admission and late EGD was defined as having EGD performed after 24 hours of admission.

Results A total of 1,789,532 subjects with UGIH were identified. Subjects who had an early EGD were less likely to have hypovolemia, acute renal failure and acute respiratory failure. On multivariable analysis, we found that subjects without EGD were 3 times more likely to die during the admission than those with early EGD. In addition, those with late EGD had $50 \%$ higher odds of dying than those with an early EGD. Also, after adjusting for all factors in the model, hospital stay was on average 3 and 3.7 days longer for subjects with no or late EGD, respectively, then for subjects with early EGD.

Conclusion Early EGD (within 24 hours) is associated with lower in-hospital mortality, morbidity, shorter length of stay and lower total hospital costs.

\section{Introduction}

Upper gastrointestinal bleeding is a common gastrointestinal emergency. Despite advances in risk factor modification and preventive strategies, upper gastrointestinal bleeding remains common, affecting up to 100 to 170 per 100,000 adults annually, with an associated mortality of $5 \%$ to $14 \%$ [1]. The hospitalization rate for upper gastrointestinal bleeding is estimated to be six-fold higher than that of lower gastrointestinal bleeding [2]. Multiple risk factors have been described for up- per gastrointestinal bleeding, including Helicobacter pylori infection, nonsteroidal anti-inflammatory medication use, chronic liver disease, and antiplatelet/anticoagulant medication use [3]. The most common causes of upper gastrointestinal bleeding include (in approximate descending order of frequency): gastric and duodenal ulcers, esophago-gastric varices, erosive esophagitis, erosive gastritis, portal gastropathy, vascular ectasia, mass lesions and Mallory-Weiss syndrome [4, 5]. In approximately $10 \%$ of cases, no source of bleeding can be identified. 
Esophagogastroduodenoscopy (EGD) has invaluable diagnostic and therapeutic utility in upper gastrointestinal bleeding. It is the modality of choice for identifying the etiology of upper gastrointestinal bleeding given its high sensitivity and specificity, along with the ability to achieve therapeutic hemostasis and prevent rebleeding in most patients [6-8]. However, the benefit of early endoscopy (EGD within 24 hours) remains unclear, with studies showing variable results. A retrospective study of a community-based practice of 909 hospitalized patients found a reduction in length of stay in all patients who underwent early endoscopy, and a reduction in the risk for recurrent bleeding and surgery in high-risk patients (those with ulcers or tears with active bleeding, arterial spurting, or a visible vessel, and a history of bleeding esophageal or gastric varices) [9]. Another observational cohort study of 3800 patients admitted with upper gastrointestinal bleeding showed similar benefits, while another population-based study found a reduction in length of stay and need for surgery [10]. One previous analysis of national inpatient data found that early EGD was associated with lower risk of mortality [11]. However, other studies did not show improved outcomes. In a retrospective analysis of 502 patients in Canada, for example, patients who underwent endoscopy within 24 hours had higher rates of mortality and need for surgery than those who underwent endoscopy $>24$ hours after presentation [12]. Another national study of over 4000 patients in the UK found no improvement in mortality or need for surgery in patients who underwent early $(<12$ hours) endoscopy, however, they did have shorter length of stay and there was a trend toward lower rebleeding rates in high-risk patients [13].

Multiple guidelines currently recommend endoscopy within 24 hours of presentation for non-variceal upper gastrointestinal bleeding [14-16] Use of early endoscopy has increased in a previous national database analysis, along with a reduction in mortality for patients hospitalized with upper gastrointestinal bleeding, however, this study did not investigate the association between early endoscopy and mortality [17]. In addition, this analysis found that despite increased rates of endoscopy over time, only $54 \%$ of patients underwent endoscopy within 24 hours in 2009. Other studies have also found that a significant proportion of patients fail to undergo endoscopy within 24 hours $[18,19]$. Hence, utilizing a nationwide database, we aimed to: 1) compare mortality during hospitalizations in those who received early EGD ( $<24$ hours) or delayed EGD (>24hours) for upper gastrointestinal bleeding, as well as those who did not undergo EGD; 2) compare the length of hospitalization, need for blood transfusion, and incidence of acute renal failure and other complications among the 3 groups; and 3) Compare the impact of early vs delayed EGD on the total costs of hospital stay in patients with upper gastrointestinal bleeding

\section{Patients and methods}

\section{Study design and data source}

This was a retrospective longitudinal study of admissions to acute care hospitals for upper gastrointestinal bleeding. Data on hospital admissions of all adult patients (18 years or older) were extracted from the National Inpatient Sample (NIS) from 2007 to 2013 . The NIS is the largest publicly available all-payer inpatient discharge database in the United States. Developed and maintained by the Agency for Healthcare Research and Quality, it comprises a $20 \%$ sample of all inpatient discharges from US hospitals meant to be representative of nationwide acute care hospitalizations. The database contains de-identified information regarding each hospitalization, including demographic characteristics, admission status, comorbidities, discharge diagnoses, procedures, outcomes, and costs of hospitalization. Patients admitted under observation status and patients admitted to short-term rehabilitation hospitals, longterm non-acute care hospitals, psychiatric hospitals, and alcoholism or chemical dependency units are not included.

\section{Study population}

We used International Classification of Diseases, Ninth Revision, Clinical Modification (ICD-9-CM) codes to identify all hospitalized adults aged at least 18 years who were discharged with a diagnosis of upper gastrointestinal bleeding, during 2007 through 2013.

The patients have listed a primary discharge diagnosis and up to 24 secondary discharge diagnoses. The patients can also have up to 15 procedure codes associated with the discharges. This database also describes the day the procedure was conducted.

Patients were classified as having upper gastrointestinal bleeding by querying all diagnostic codes for the ICD-9-CM codes corresponding to upper gastrointestinal bleeding. For these patients, performance of EGD during admission was determined by querying all procedural codes for the ICD-9-CM codes corresponding to EGD; early EGD was defined as having EGD performed within 24 hours of admission and late EGD was defined as having EGD performed after 24 hours of admission. Patients with multiple EGDs during the same admission were classified based on when the first procedure was done. All diagnostic and procedural codes used for classifications are found in Appendix 1.

\section{Definition of Variables, comorbidities, and other covariates}

The NIS contains demographic information on all hospitalizations, including age, gender, race and, primary and secondary insurance. Patient's comorbidity was adjusted. The Healthcare Cost and Utilization Project (HCUP) Comorbidity Software was used to generate Elixhauser comorbidities from ICD-9 CM diagnosis codes (https://www.hcup-us.ahrq.gov/toolssoftware/comorbidity/comorbidity.jsp). A modified Charlson Comorbidity Index $(\mathrm{CCI})$ was calculated using the NIS Disease Severity Measure files. Several modifications were performed because not all 22 comorbidities are coded into the NIS database: 1) history of myocardial infarction was omitted and 2) liver disease was given an adjusted weight of 2 points rather than 1 for mild disease and 3 points for moderate to severe disease.

Apart from the comorbidities scored by the $\mathrm{CCl}$, we also identified the presence of specific comorbidities that may play an important role in the severity of bleeding. Indicators of se- 
verity of bleeding such as hypovolemia/shock, acute renal failure and need for dialysis, respiratory failure, as well as the frequency of various supportive interventions as surrogate indicators of hemodynamic status, such as endotracheal intubation, were also noted and used as covariates in multivariate analysis of the outcomes by using the ICD-9-CM codes.

\section{Outcomes}

We analyzed the following outcomes: 1) prevalence of upper gastrointestinal bleeding in hospitalizations, 2) timing of endoscopy in admissions associated with upper gastrointestinal bleeding, 3) in-hospital mortality in admissions with upper gastrointestinal bleeding in respect to timing of endoscopy; and 4) Length of stay and hospitalization charges associated with upper gastrointestinal bleeding in respect to timing of endoscopy.

\section{Statistical analysis}

Data are presented as mean \pm standard deviation for continuous variables or weighted frequency (\%) for categorical factors. A univariate analysis was performed to assess differences between the 3 groups (no EGD, early EGD and late EGD); continuous variables were compared using $t$-tests and categorical variables were compared using Rao-Scott chi-square tests. In addition, multivariable analysis was performed to assess differences between the groups in terms of the outcomes of interest while adjusting for patient and hospital characteristics. Logistic regression analysis was used to model mortality and linear regression analysis was used for length of stay and total costs. NIS is based on a complex sampling design that includes stratification, clustering and weighting; SAS Survey procedures facilitate the unbiased assessment of population estimates. A $P<$ 0.001 was considered statistically significant because of the large sample size; this significance criterion has been used by previous NIS studies. All analyses were performed using SAS (version 9.4, The SAS Institute, Cary, NC). $\checkmark$ Table 1 Etiology of upper gastrointestinal hemorrhage.

\begin{tabular}{|l|c|}
\hline Upper gastrointestinal hemorrhage & $\mathbf{n}(\%)$ \\
\hline Bleeding esophageal varices & $222,148(12.4)$ \\
\hline Bleeding peptic ulcer & $876,320(49.0)$ \\
\hline Mallory-Weiss & $186,634(10.4)$ \\
\hline Gastritis with hemorrhage & $423,450(23.7)$ \\
\hline Dieulafoy's lesion of stomach or duodenum & $32,790(1.8)$ \\
\hline $\begin{array}{l}\text { Angiodysplasia of stomach or duodenum } \\
\text { with hemorrhage }\end{array}$ & $155,594(8.7)$ \\
\hline
\end{tabular}

\section{Results}

From the NIS database dated 2007 to 2013, a total of 2,066,707 adult patients (older than 18 years of age) were identified as having the primary discharge diagnosis of upper gastrointestinal bleeding. Among these patients, 1,735,116 (83.96\%) had undergone EGD during the admission, while 331,591 (16\%) did not have an EGD. Of the patients who underwent EGD, $1,020,744$ were noted to have had an early EGD (within the first 24 hours), while 714,372 had delayed EGD (>24 hours).

- Table 1 presents the various etiologies of upper gastrointestinal bleeding among the hospital admissions. Bleeding peptic ulcer was the by far the most common cause of upper gastrointestinal bleeding making up almost $50 \%$ of the patient population. Much less common causes of upper gastrointestinal bleeding, such as Dieulafoy lesions, comprised $1.9 \%$ of the studied subjects.

- Table 2 shows the basic characteristics and demographics of the 3 studied groups (early EGD, delayed EGD, no EGD). Patients in the delayed EGD group were older with a mean age of $67.6 \pm 0.11$ years, compared to $64.0 \pm 0.09$ years in the early EGD group and $63.9 \pm 0.13$ years in the no EGD group $(P<$ $0.001)$. Patients in all groups were more likely to be men and

- Table 2 Patient and hospital characteristics.

\begin{tabular}{|l|l|l|l|}
\hline Factor & $\begin{array}{l}\text { Early EGD } \\
\mathbf{n = 8 7 0 , 1 5 9}\end{array}$ & $\begin{array}{l}\text { Late EGD } \\
\mathbf{n = 6 3 1 , 4 1 2}\end{array}$ & $\begin{array}{l}\text { No EGD } \\
\mathbf{n = 2 8 7 , 9 6 1}\end{array}$ \\
\hline Age (years), mean \pm SD & $64.0 \pm 0.10$ & $67.6 \pm 0.12$ & $64.1 \pm 0.15$ \\
\hline Age (years) & & $24,095(3.8)$ & $24,620(8.5)$ \\
\hline $18-35$ & $49,577(5.7)$ & $71,031(11.2)$ & $43,509(15.1)$ \\
\hline $36-50$ & $135,750(15.6)$ & $161,303(25.5)$ & $73,123(25.4)$ \\
\hline $51-65$ & $261,147(30.0)$ & $374,983(59.4)$ & $146,709(50.9)$ \\
\hline$>65$ & $423,686(48.7)$ & & $151,710(52.7)$ \\
\hline Gender & & $326,560(51.7)$ & $136,167(47.3)$ \\
\hline Male & $503,200(57.8)$ & $304,828(48.3)$ & \\
\hline Female & $366,816(42.2)$ & & $170,051(59.1)$ \\
\hline Race & & $345,664(54.7)$ & $<0.001$ \\
\hline White & $564,485(64.9)$ & \\
\hline
\end{tabular}


Table 2 (Continuation)

\begin{tabular}{|c|c|c|c|c|}
\hline Factor & $\begin{array}{l}\text { Early EGD } \\
n=870,159\end{array}$ & $\begin{array}{l}\text { Late EGD } \\
n=631,412\end{array}$ & $\begin{array}{l}\text { No EGD } \\
n=287,961\end{array}$ & $P$ value \\
\hline Black & $92,057(10.6)$ & $72,628(11.5)$ & $36,626(12.7)$ & \\
\hline Hispanic & $82,140(9.4)$ & $46,547(7.4)$ & $22,135(7.7)$ & \\
\hline Other & $54,097(6.2)$ & $30,317(4.8)$ & $14,699(5.1)$ & \\
\hline Unknown & $77,380(8.9)$ & $136,256(21.6)$ & $44,450(15.4)$ & \\
\hline Insurance & & & & $<0.001$ \\
\hline Medicare & $457,208(52.7)$ & $405,556(64.3)$ & $165,366(57.6)$ & \\
\hline Medicaid & $85,399(9.8)$ & $58,067(9.2)$ & $35,398(12.3)$ & \\
\hline Private Insurance & $218,286(25.1)$ & $115,434(18.3)$ & $55,025(19.2)$ & \\
\hline Other & $107,152(12.3)$ & $51,319(8.1)$ & $31,362(10.9)$ & \\
\hline $\mathrm{CCl}$, mean $\pm \mathrm{SD}$ & $3.5 \pm 0.01$ & $4.2 \pm 0.02$ & $3.9 \pm 0.02$ & $<0.001$ \\
\hline $\mathrm{CCl}$ & & & & $<0.001$ \\
\hline 0 & $98,254(11.3)$ & $40,975(6.5)$ & $31,234(10.8)$ & \\
\hline 1 & $97,742(11.2)$ & $46,192(7.3)$ & $25,215(8.8)$ & \\
\hline 2 & $117,390(13.5)$ & $65,663(10.4)$ & $32,524(11.3)$ & \\
\hline 3 & $136,029(15.6)$ & 87,965 (13.9) & $37,447(13.0)$ & \\
\hline $4+$ & $420,744(48.4)$ & 390,617 (61.9) & $161,542(56.1)$ & \\
\hline Weekend admission & & & & $<0.001$ \\
\hline Weekday & $684,462(78.7)$ & $463,964(73.5)$ & $220,873(76.7)$ & \\
\hline Weekend & $185,698(21.3)$ & $167,448(26.5)$ & $67,088(23.3)$ & \\
\hline Hospital location & & & & $<0.001$ \\
\hline Rural & $91,210(10.6)$ & $75,349(12.0)$ & $46,290(16.2)$ & \\
\hline Urban & $771,664(89.4)$ & $552,410(88.0)$ & $239,666(83.8)$ & \\
\hline Hospital teaching status & & & & 0.066 \\
\hline Non-teaching & $506,008(58.6)$ & $357,462(56.9)$ & $162,213(56.7)$ & \\
\hline Teaching & $356,865(41.4)$ & $270,297(43.1)$ & $123,743(43.3)$ & \\
\hline Bed size of hospital & & & & $<0.001$ \\
\hline Small & $94,952(11.0)$ & $71,213(11.3)$ & $40,098(14.0)$ & \\
\hline Medium & $225,847(26.2)$ & $149,910(23.9)$ & $69,256(24.2)$ & \\
\hline Large & $542,074(62.8)$ & $406,636(64.8)$ & $176,602(61.8)$ & \\
\hline Region of hospital & & & & $<0.001$ \\
\hline Northeast & $156,660(18.0)$ & $106,811(16.9)$ & $54,506(18.9)$ & \\
\hline Midwest & $169,273(19.5)$ & $174,267(27.6)$ & $62,823(21.8)$ & \\
\hline South & $345,145(39.7)$ & $244,930(38.8)$ & $113,465(39.4)$ & \\
\hline West & 199,081 (22.9) & $105,404(16.7)$ & $57,166(19.9)$ & \\
\hline Median household income & & & & $<0.001$ \\
\hline 1 & $234,860(27.6)$ & $189,671(30.8)$ & $90,823(32.4)$ & \\
\hline 2 & $225,128(26.5)$ & $165,045(26.8)$ & $74,592(26.6)$ & \\
\hline 3 & $206,899(24.3)$ & $145,666(23.6)$ & $63,398(22.6)$ & \\
\hline 4 & $182,937(21.5)$ & $115,635(18.8)$ & $51,602(18.4)$ & \\
\hline
\end{tabular}


- Table 3 Adverse events and outcomes.

\begin{tabular}{|l|l|l|l|}
\hline Factor & $\begin{array}{l}\text { Early EGD } \\
\mathbf{n = 8 7 0 , 1 5 9}\end{array}$ & $\begin{array}{l}\text { Late EGD } \\
\mathbf{n = 6 3 1 , 4 1 2}\end{array}$ & $\begin{array}{l}\text { No EGD } \\
\mathbf{n = 2 8 7 , 9 6 1}\end{array}$ \\
\hline Blood transfusion & $477,264(54.8)$ & $323,938(51.3)$ & $97,391(33.8)$ \\
\hline Hypovolemia/shock & $116,826(13.4)$ & $88,880(14.1)$ & $62,538(14.8)$ \\
\hline Acute renal failure & $139,094(16.0)$ & $164,278(26.0)$ & $30,886(10.7)$ \\
\hline Acute respiratory failure & $43,479(5.0)$ & $51,071(8.1)$ & - \\
\hline Endoscopic therapy & $375,649(43.2)$ & $195,296(30.9)$ & $7.7 \pm 0.09$ \\
\hline LOS (days) & $4.6 \pm 0.02$ & $8.5 \pm 0.08$ & $62266.7 \pm 1737.1$ \\
\hline Total charges (\$) & $38313.6 \pm 529.8$ & $64412.2 \pm 1267.2$ & $58987.4 \pm 1653.7$ \\
\hline Inflation-adjusted charges (2013\$) & $36347.6 \pm 502.3$ & $60915.2 \pm 1202.6$ & $24,491(8.5)$ \\
\hline Died during hospitalization & $26,181(3.0)$ & $26,457(4.2)$ & $<0.001$ \\
\hline Data presented as mean \pm standard error or Weighted Frequency (\%) & & $<0.001$ \\
\hline
\end{tabular}

to have Medicare. Patients who underwent delayed EGD had a higher $\mathrm{CCl}$ than the other two groups $(P<0.001)$. The proportion of patients with a $\mathrm{CCl}$ of 3 or more in the delayed group was $76 \%$ as compared to $61.4 \%$ in the early EGD group and $69.1 \%$ in the no EGD group $(P<0.001)$.

The rates of adverse events (AEs) also varied among the groups as shown in $>$ Table 3 . Endoscopic therapy was found to be more common in patients who underwent early EGD (43.8\%) compared to the delayed EGD group (31.6\%) $(P<$ $0.001)$. Patients who did not undergo EGD were less likely to get blood transfusions (33.7\%) as compared to those who underwent early EGD (55.0\%) and delayed EGD (51.6\%). Patients who underwent early EGD were less likely to have hypovolemia and shock than the other groups (13.7\% in the early EGD group, with $14.2 \%$ and $14.9 \%$ in the delayed and no-EGD groups respectively). Acute respiratory failure was more likely in the no-EGD group at $10.9 \%$, than $8.3 \%$ in the delayed EGD group and $5.2 \%$ in the early EGD group $(P<0.001)$. Patients in the delayed EGD group were more likely to develop acute renal failure (27.0\%) as compared with the no-EGD group (24.5\%) and the early EGD group $(16.7 \%)(P<0.001)$.

\section{Outcomes}

Mortality

The primary outcome of the study was in-hospital mortality. Mortality was found to be significantly higher in the delayed EGD group as compared to the early EGD group (4.2\% vs $3.0 \%$ $P<0.001)$ ( $>$ Table 3 ). Mortality was much higher in the noEGD group ( $8.5 \%$ ) compared to the early and late EGD groups $(P<0.001)$.

On multivariate analysis, patients who did not undergo EGD were 3 times likely to die compared to those who underwent early EGD (OR 3.0; 99.9\% Cl 2.8-3.2; P<0.001) ( Table 4). Also, those who had a delayed EGD were 1.4 times more likely to die compared with early EGD patients (OR $1.4 ; 99.9 \% \mathrm{CI}$ $1.3-1.5 ; P<0.001)$. Patients with a $\mathrm{CCl}$ of 4 or more had an over 4-fold chance of mortality compared to those with a $\mathrm{CCI}$ of 0 (OR $4.4 ; 99.9 \% \mathrm{Cl} 3.7-5.1 ; P<0.001)$. Other risk factors associated with a higher in-hospital mortality for upper gastrointestinal bleeding on multivariate analysis included male sex (OR 1.4; 99.9\% Cl 1.3-1.5; $P<0.001$ ), Hispanic ethnicity (OR $1.1 ; 99.9 \% \mathrm{Cl} 1.0-1.2 ; P<0.001)$, insurance other than Medicare (OR 1.4; 99.9\% Cl 1.2-1.6; $P<0.001)$, weekend admission (OR $1.1 ; 99.9 \% \mathrm{Cl} 1.0-1.2 ; P<0.001$ ), and admission to a large (OR 1.3; 99.9\% Cl 1.2 - 1.5; $\mathrm{p}<0.001)$, urban (OR 1.3; $99.9 \% \mathrm{Cl}$ $1.1-1.5 ; P<0.001)$ teaching hospital (OR $1.4 ; 99.9 \% \mathrm{Cl} 1.2-$ $1.4 ; P<0.001)$. Interestingly, black race was associated with a lower risk for in-hospital mortality (OR 0.9; $99.9 \% \mathrm{Cl} 0.8-0.9$; $P<0.001)$.

\section{Length of hospital stay}

Length of hospital stay was found to vary among the three groups as well. Patients who underwent early EGD had a shorter length of stay compared to the delayed EGD and no-EGD groups (4.6 vs 8.5 vs 7.6 days, respectively; $P<0.001$ ) ( $>$ Table 3 ) On multivariate analysis, hospital stay was 2.9 days longer for patients who did not undergo EGD compared to patients who underwent early EGD $(99.9 \% \mathrm{Cl} 2.7-3.2 ; P<0.001)$. Patients who underwent delayed EGD were in the hospital for 3.7 days longer compared to early EGD patients $(99.9 \% \mathrm{Cl} 3.5-4.0$; $P<0.001)$. Severity of upper gastrointestinal bleeding as determined by the $\mathrm{CCl}$ score was found to be a predictor of length of stay. A $\mathrm{CCl}$ score of 1 was associated with 1.3-day increase in length of stay $(99.9 \% \mathrm{Cl} 1.08-1.5 P<0.001)$. Additional $\mathrm{CCl}$ points were associated with a gradual increase in length of hospital stay. Reaching a CCl of 4 was an associated 3.8-day increase in length of stay $(99.9 \% \mathrm{Cl} 3.5-4.1 ; P<0.001)$ as compared to a $\mathrm{CCl}$ of 0 ( $>$ Table 5).

\section{Hospital cost}

The increase in mean hospital costs correlated with increasing disease severity as measured by the $\mathrm{CCl}$, as well as delayed EGD compared to early EGD. Total charges were significantly higher in the delayed EGD group and no-EGD group compared to the early EGD group $(\$ 66775.5 \pm 1143.8$ vs $\$ 64023.9 \pm 1561.8$ vs 
- Table4 Multivariable analysis for predictors of in-hospital mortality in patients with upper gastrointestinal hemorrhage.

\begin{tabular}{|c|c|c|}
\hline Factor & OR $(99.9 \% \mathrm{Cl})$ & $P$ value \\
\hline \multicolumn{3}{|l|}{ EGD } \\
\hline No vs. early & $3.0(2.8,3.2)$ & $<0.001$ \\
\hline Late vs. early & $1.4(1.3,1.5)$ & $<0.001$ \\
\hline Age (5 yr. increase) & $0.99(0.97,1.00)$ & 0.010 \\
\hline Male vs. female & $1.4(1.3,1.5)$ & $<0.001$ \\
\hline \multicolumn{3}{|l|}{ Race } \\
\hline Black vs. white & $0.86(0.78,0.95)$ & $<0.001$ \\
\hline Hispanic vs. white & $1.1(1.00,1.2)$ & $<0.001$ \\
\hline Other vs. white & $1.02(0.89,1.2)$ & 0.24 \\
\hline Unknown vs. white & $0.93(0.82,1.05)$ & 0.070 \\
\hline \multicolumn{3}{|l|}{ Insurance } \\
\hline Medicaid vs. medicare & $1.7(1.5,1.9)$ & $<0.001$ \\
\hline Private Insurance vs. medicare & $1.1(1.04,1.2)$ & $<0.001$ \\
\hline Other vs. medicare & $1.4(1.2,1.6)$ & $<0.001$ \\
\hline \multicolumn{3}{|l|}{$\mathrm{CCl}$} \\
\hline 1 vs. 0 & $1.6(1.4,1.9)$ & $<0.001$ \\
\hline 2 vs. 0 & $2.4(2.0,2.8)$ & $<0.001$ \\
\hline 3 vs. 0 & $3.0(2.5,3.6)$ & $<0.001$ \\
\hline $4+v s .0$ & $4.2(3.6,5.0)$ & $<0.001$ \\
\hline Weekend vs. weekday & $1.08(1.01,1.2)$ & $<0.001$ \\
\hline Urban vs. rural hospital & $1.3(1.1,1.5)$ & $<0.001$ \\
\hline Teaching vs. non-teaching hospital & $1.3(1.2,1.4)$ & $<0.001$ \\
\hline \multicolumn{3}{|l|}{ Bed size of hospital } \\
\hline Medium vs. small & $1.1(1.00,1.3)$ & 0.93 \\
\hline Large vs. small & $1.3(1.2,1.5)$ & $<0.001$ \\
\hline \multicolumn{3}{|l|}{ Region of hospital } \\
\hline Midwest vs. northeast & $0.83(0.73,0.95)$ & $<0.001$ \\
\hline South vs. northeast & $0.93(0.83,1.04)$ & 0.68 \\
\hline West vs. northeast & $0.99(0.87,1.1)$ & 0.009 \\
\hline \multicolumn{3}{|l|}{ Median household income } \\
\hline 2 vs. 1 & $0.95(0.88,1.03)$ & 0.73 \\
\hline 3 vs. 1 & $0.93(0.85,1.01)$ & 0.27 \\
\hline 4 vs. 1 & $0.90(0.82,1.00)$ & 0.018 \\
\hline
\end{tabular}

$\mathrm{OR}$, odds ratio; $\mathrm{Cl}$, confidence interval

$\$ 39608.2 \pm 464.2 P<0.001)$ ( $>$ Table 3). In multivariate analysis, cost of hospital stay (inflation-adjusted hospital charges) was found to be $\$ 28,024.62$ higher for delayed EGD $(99.9 \% \mathrm{Cl}$ $25,077.42-30,971.81 P<0.001)$ and $\$ 24,780.18$ higher for the no-EGD group $(99.9 \% \mathrm{Cl} 20,725.18-28,835.17 p<0.001)$
- Table 5 Multivariable analysis for predictors of length of stay in patients with upper gastrointestinal hemorrhage.

\begin{tabular}{|c|c|c|}
\hline Factor & Estimate $(99.9 \% \mathrm{Cl})$ & $P$ value \\
\hline \multicolumn{3}{|l|}{ EGD } \\
\hline No vs. early & $3.0(2.7,3.3)$ & $<0.001$ \\
\hline Late vs. early & $3.7(3.5,4.0)$ & $<0.001$ \\
\hline Age (5 yr. increase) & $-0.18(-0.22,-0.14)$ & $<0.001$ \\
\hline Male vs. female & $0.36(0.27,0.46)$ & $<0.001$ \\
\hline \multicolumn{3}{|l|}{ Race } \\
\hline Black vs. white & $0.30(0.05,0.55)$ & $<0.001$ \\
\hline Hispanic vs. white & $-0.03(-0.32,0.27)$ & 0.77 \\
\hline Other vs. white & $0.42(0.12,0.73)$ & $<0.001$ \\
\hline Unknown vs. white & $-0.78(-1.2,-0.41)$ & $<0.001$ \\
\hline \multicolumn{3}{|l|}{ Insurance } \\
\hline Medicaid vs. medicare & $0.92(0.65,1.2)$ & $<0.001$ \\
\hline Private Insurance vs. medicare & $-0.28(-0.45,-0.11)$ & $<0.001$ \\
\hline Other vs. medicare & $-0.63(-0.84,-0.41)$ & $<0.001$ \\
\hline \multicolumn{3}{|l|}{$\mathrm{CCl}$} \\
\hline 1 vs. 0 & $1.2(1.02,1.5)$ & $<0.001$ \\
\hline 2 vs. 0 & $2.0(1.8,2.3)$ & $<0.001$ \\
\hline 3 vs. 0 & $2.7(2.4,3.0)$ & $<0.001$ \\
\hline $4+$ vs. 0 & $3.8(3.4,4.1)$ & $<0.001$ \\
\hline Weekend vs. weekday & $-0.26(-0.37,-0.15)$ & $<0.001$ \\
\hline Urban vs. rural hospital & $1.4(1.1,1.6)$ & $<0.001$ \\
\hline $\begin{array}{l}\text { Teaching vs. non-teaching } \\
\text { hospital }\end{array}$ & $0.97(0.68,1.3)$ & $<0.001$ \\
\hline \multicolumn{3}{|l|}{ Bed size of hospital } \\
\hline Medium vs. small & $0.17(-0.18,0.52)$ & 0.11 \\
\hline Large vs. small & $0.92(0.55,1.3)$ & $<0.001$ \\
\hline \multicolumn{3}{|l|}{ Region of hospital } \\
\hline Midwest vs. northeast & $-1.1(-1.5,-0.68)$ & $<0.001$ \\
\hline South vs. northeast & $-0.49(-0.85,-0.13)$ & $<0.001$ \\
\hline West vs. Northeast & $-0.86(-1.3,-0.43)$ & $<0.001$ \\
\hline \multicolumn{3}{|l|}{ Median household income } \\
\hline 2 vs. 1 & $-0.00(-0.17,0.16)$ & 0.94 \\
\hline 3 vs. 1 & $-0.12(-0.32,0.08)$ & 0.044 \\
\hline 4 vs. 1 & $-0.11(-0.36,0.13)$ & 0.12 \\
\hline
\end{tabular}

when compared with the early EGD group ( $\bullet$ Table 6$)$. Cost of stay was also impacted by the severity of upper gastrointestinal bleeding. A CCl score of 1 was associated with a \$14801.58 increase in the cost of hospital stay as compared to a $\mathrm{CCl}$ score of 0 
- Table6 Multivariable analysis for predictors of inflation-adjusted hospital charges in patients with upper gastrointestinal hemorrhage.

\begin{tabular}{|c|c|c|}
\hline Factor & Estimate $(99.9 \% \mathrm{Cl})$ & $P$ value \\
\hline \multicolumn{3}{|l|}{ EGD } \\
\hline No vs. early & $24,378.86(19,943.49,28,814.23)$ & $<0.001$ \\
\hline Late vs. early & $26,852.55(23,601.21,30,103.90)$ & $<0.001$ \\
\hline Age (5 yr. increase) & $-3,022.01(-3,613.88,-2,430.14)$ & $<0.001$ \\
\hline Male vs. female & $5,513.55(4,457.10,6,570.00)$ & $<0.001$ \\
\hline \multicolumn{3}{|l|}{ Race } \\
\hline Black vs. white & $1,631.11(-2,274.41,5,536.62)$ & 0.17 \\
\hline Hispanic vs. white & $6,284.29(1,389.01,11,179.57)$ & $<0.001$ \\
\hline Other vs. white & $6,413.02(2,175.14,10,650.90)$ & $<0.001$ \\
\hline Unknown vs. white & $-12,386.24(-16,988.51,-7,783.97)$ & $<0.001$ \\
\hline \multicolumn{3}{|l|}{ Insurance } \\
\hline Medicaid vs. medicare & $6,987.58(3,962.04,10,013.12)$ & $<0.001$ \\
\hline Private insurance vs. medicare & $-8.59(-2,079.90,2,062.73)$ & 0.99 \\
\hline Other vs. medicare & $-6,357.65(-9,166.05,-3,549.26)$ & $<0.001$ \\
\hline \multicolumn{3}{|l|}{$\mathrm{CCl}$} \\
\hline 1 vs. 0 & $13,534.73(11,216.53,15,852.93)$ & $<0.001$ \\
\hline 2 vs. 0 & $23,681.27(20,222.78,27,139.77)$ & $<0.001$ \\
\hline 3 vs. 0 & $31,516.04(27,386.09,35,646.00)$ & $<0.001$ \\
\hline $4+$ vs. 0 & $39,525.91(34,474.41,44,577.41)$ & $<0.001$ \\
\hline Weekend vs. weekday & $-1,469.50(-2,663.68,-275.31)$ & $<0.001$ \\
\hline Urban vs. rural hospital & $18-682.78(15,062.53,22,303.03)$ & $<0.001$ \\
\hline Teaching vs. non-teaching hospital & $11,516.06(6,135.51,16,896.61)$ & $<0.001$ \\
\hline \multicolumn{3}{|l|}{ Bed size of hospital } \\
\hline Medium vs. small & $4,670.46(-323.05,9,663.97)$ & 0.002 \\
\hline Large vs. small & $15,343.59(9,701.29,20,985.88)$ & $<0.001$ \\
\hline \multicolumn{3}{|l|}{ Region of hospital } \\
\hline Midwest vs. northeast & $-12,299.10(-22,791.04,-1,807.16)$ & $<0.001$ \\
\hline South vs. northeast & $-8,473.23(-18,701.45,1,754.99)$ & 0.006 \\
\hline West vs. northeast & $10,772.76(-274.86,21,820.39)$ & 0.001 \\
\hline \multicolumn{3}{|l|}{ Median household income } \\
\hline 2 vs. 1 & $387.45(-2,043.14,2,818.05)$ & 0.60 \\
\hline 3 vs. 1 & $1,774.22(-1,751.15,5,299.58)$ & 0.098 \\
\hline 4 vs. 1 & $2,724.03(-2,612.88,8,060.93)$ & 0.093 \\
\hline
\end{tabular}

(99\% Cl $12465.57-17137.58 P<0.001)$, while a CCl score of 4 was associated with a $\$ 41,782.41$ increase in cost of hospitalization compared to a CCI score of 0 . (Cl 37,252.13-46,312.69 $P<0.001)$

\section{Discussion}

This nationwide inpatient database study shows that early EGD was associated with lower morbidity and mortality as compared to delayed EGD or no EGD. Patients who underwent early EGD had lower incidence of acute renal and respiratory failure. 
In addition, the overall costs, including the costs of the procedure, and length of hospital stay were much higher in patients who did not undergo early EGD.

Acute upper gastrointestinal bleeding is a true gastrointestinal emergency [20]. Previous data found mortality associated with upper gastrointestinal bleeding ranging from $3.5 \%$ to $10 \%$ [15]. However, analysis of more recent nationwide data found the mortality associated with upper gastrointestinal bleeding has been decreasing over the past 2 decades, down to $2.1 \%$ in 2009 [17]. Multiple guidelines recommend endoscopy within 24 hours for patients with upper gastrointestinal bleeding [14-16]. Endoscopic intervention in patients with upper gastrointestinal bleeding is associated with a reduction in blood transfusion requirements, length of intensive care unit stay, and total hospital stay [15]. A nationwide database analysis found that rates of early EGD have been increasing over the past 20 years [17].

Our study found a significant reduction in mortality associated with early EGD as compared to delayed or no EGD. A previous nationwide analysis found reduction in mortality in concordance with an increase in early EGD in upper gastrointestinal bleeding over the past 20 years leading up until 2009 [17]. In addition, another analysis of nationwide data prior to 2007 also found a reduction in mortality with early EGD [11]. Our study adds to these results by including more recent inpatient data, and controlling for other risk factors using logistic regression to detect the association of lower mortality with early EGD, as compared to delayed EGD or no EGD. Performing early EGD may be associated with lower mortality rates for multiple reasons, including earlier time to endoscopic therapies to control active bleeding and subsequently reduce need for transfusions and other supportive therapies, such as vasopressors, and risk stratification of high-risk and low-risk lesions, which may triage patients to either more aggressive interventions in high-risk lesions or earlier discharge in low-risk lesions.

Our study also found a lower rate of complications, such as renal failure and respiratory failure, in patients who underwent early EGD. Early EGD has been previously found to reduce the risk of recurrent bleeding, transfusion requirements [21] and the need for emergent surgery especially in patients with high-risk stigmata for upper gastrointestinal bleeding [9]. Cooper et al have also shown that early endoscopy is associated with a clinically significant, although not statistically significant, lower risk of recurrent bleeding after adjusting for confounding factors [9].

Early EGD also has benefits in reducing length of stay and hospital costs. In our analysis, early EGD was associated with a reduction by over 3 days of hospitalization compared to both delayed and no EGD. This may be for several reasons, however likely bleeding lesions may be effectively treated and stopped sooner, leading to faster recovery and low-risk lesions may be identified sooner, leading to earlier discharge. This in turn can lead to an overall reduction in costs of the associated hospitalization. This is consistent with previous studies that have found a reduction in length of stay with early EGD [21,22].

Previous studies have found that EGD can help in deciding if the patient has low-risk stigmata for recurrent bleeding and thus helps in deciding if we can safely discharge a patient home with follow up as an outpatient [15]. In a study by Cipolletta et al [23], 464 patients who had acute upper gastrointestinal bleeding underwent EGD within 12 hours of hospital admission, of whom 95 patients were randomized to outpatient versus inpatient management. No difference in the clinical outcome was noted between the 2 groups. Thus, this study helps delineate the role of early EGD in deciding outpatient versus inpatient management. In addition, in this study, the median cost was $\$ 340$ in the outpatient group versus $\$ 3940$ in the inpatient group [23]. Lai et al have also shown this in their retrospective study in which patients with clean base ulcer were stable for discharge on the day of procedure, without an increased risk for rebleeding [24]. Based on these results, early EGD seems to help in reducing the substantial burden imposed on health care resources without adversely affecting the patient outcome.

In addition to reducing inpatient costs, early EGD also reduces costs in the outpatient arena. This has been also been reported by Lee et al, where they showed that a patient population who underwent early endoscopy had lesser post discharge unplanned physician visits [25].

Although diagnostic endoscopies and endoscopic therapies have become more frequently utilized in patients who present with upper gastrointestinal bleeding, the timing of EGD varies significantly. Previous studies have found improved rates of early endoscopy, however, al bare majority of patients receive EGD within 24 hours [17-19]. In our study, only $59 \%$ of patients who received an EGD had it within the first 24 hours. Considering the benefits of early EGD, protocols should be set regarding the timing of EGD in patients with acute non-variceal upper gastrointestinal bleeding, as many a times, timing alone often dictates clinical success. Early EGD provides the benefit of achieving early hemostasis in high-risk patients whereas lowrisk patients benefit by avoiding prolonged hospitalization. Similarly, it has been shown that late EGD offers no additional benefit over standard medical management [21]. Various reasons exist for why patients may not receive EGD within 24 hours, especially in a large database analysis such as our study, including weekend admissions, incorrect diagnoses, and patients who may have been classified as low enough risk to not require $E G D$. There may also be patient-related factors that could delay EGD, specifically certain co-morbidities, such as severe cardiopulmonary instability or coagulopathy, which may be unavoidable and require correction prior to EGD. Lastly, certain disease factors in upper gastrointestinal bleeding may necessitate radiographic or surgical intervention preferentially to EGD. Overall, a protocol-based approach for upper gastrointestinal bleeding stressing early EGD, with establishment of a gastrointestinal rapid response team available after hours and on weekends, may not only be cost-effective but also better for patient safety and outcomes.

Our study has several strengths, in that it included a large sample size of representative hospital admissions for the United States over a recent period of time (2007-2013), and thus should largely reflect current practice. However, in interpreting the findings of our study, several potential limitations should be kept in mind. First, the study wasbased on ICD-9 CM discharge 
diagnoses, so there is a possibility of incorrect classification. Second, the study was based on discharge diagnosis, and information on the endoscopic findings was not available. Hence, we could not separate the patients into low-risk and high-risk categories based on stigmata of recent hemorrhage. Third, our study was based on hospitalized patients. It did not reflect patients who underwent outpatient endoscopy for upper gastrointestinal bleeding that might have prevented hospitalization.

Lastly, a significant potential limitation is the retrospective nature of the study, and given that, we can only report the association of early EGD with improved outcomes and we cannot infer causality. Specifically, there exists the potential for confounding factors in the delayed and no-EGD groups that led them to have either delayed or no EGD. As mentioned above, these patients could have had underlying co-morbidities, such as severe cardiopulmonary instability or coagulopathy, preventing them from undergoing EGD in a timely manner, or they might have had severe or complicated disease requiring alternative therapies be performed such as angiography, TIPS or surgery. In that case, poor outcomes may have been related to the underlying severity of illness, as opposed to lack of intervention. Alternatively, patients could have had very mild upper gastrointestinal bleeding that did not warrant an EGD. We were unable to adjudicate whether these 2 groups balanced each other out given the retrospective nature of our analysis, thus the possibility exists that the higher rate of AEs in the delayed EGD group were not in fact related to a lack of intervention, but to a patient's underlying disease or comorbidities. Due to these limitations, further large-scale retrospective and, more importantly, prospective studies should be undertaken in order to assess outcomes based on timing of EGD, ideally in similarly matched patient groups based on clinical status, underlying comorbidities, and whether their gastrointestinal bleeding was severe or complicated such that it may have required radiographic or surgical intervention.

\section{Conclusions}

Early EGD (within 24 hours) is associated with lower in-hospital mortality and morbidity as compared to delayed EGD or no EGD in-hospital admissions for upper gastrointestinal bleeding. In addition, early EGD is associated with shorter length of stay and lower total hospital costs. Despite multiple guidelines recommending early EGD, the rate of early EGD remains inadequate. Future protocols should be developed to incorporate early EGD in order to improve patient, hospital, and economic outcomes. Given this study is retrospective, we cannot determine that early EGD was the causal driver of improved outcomes, thus future prospective studies will be required to confirm this finding.

\section{Competing interests}

None
References

[1] Colle I, Wilmer A, Le Moine O et al. Upper gastrointestinal tract bleeding management: Belgian guidelines for adults and children. Acta Gastroenterol Belg 2011; 74: 45-66

[2] Longstreth GF. Epidemiology of hospitalization for acute upper gastrointestinal hemorrhage: a population-based study. Am J Gastroenterol 1995; 90: 206-210

[3] Tielleman T, Bujanda D, Cryer B. Epidemiology and risk factors for upper gastrointestinal bleeding. Gastrointest Endosc Clin N Am 2015; 25: $415-428$

[4] Enestvedt BK, Gralnek IM, Mattek N et al. An evaluation of endoscopic indications and findings related to nonvariceal upper-GI hemorrhage in a large multicenter consortium. Gastrointest Endosc 2008; 67: $422-429$

[5] Wollenman CS, Chason R, Reisch JS et al. Impact of ethnicity in upper gastrointestinal hemorrhage. J Clin Gastroenterol 2014; 48: 343 - 350

[6] Biecker E. Diagnosis and therapy of non-variceal upper gastrointestinal bleeding. World J Gastrointest Pharmacol Ther 2015; 6: 172-182

[7] Kaliamurthy M, Lee MG, Mills M et al. Upper gastrointestinal bleeding: a Jamaican perspective. West Indian Med J 2011; 60: 289-292

[8] Meltzer AC, Klein JC. Upper gastrointestinal bleeding: patient presentation, risk stratification, and early management. Gastroenterol Clin North Am 2014; 43: 665-675

[9] Cooper GS, Chak A, Way LE et al. Early endoscopy in upper gastrointestinal hemorrhage: associations with recurrent bleeding, surgery, and length of hospital stay. Gastrointest Endosc 1999; 49: 145-152

[10] Cooper GS, Kou TD, Wong RC. Use and impact of early endoscopy in elderly patients with peptic ulcer hemorrhage: a population-based analysis. Gastrointest Endosc 2009; 70: 229-235

[11] Wysocki JD, Srivastav S, Winstead NS. A nationwide analysis of risk factors for mortality and time to endoscopy in upper gastrointestinal haemorrhage. Aliment Pharmacol Ther 2012; 36: 30-36

[12] Sarin N, Monga N, Adams PC. Time to endoscopy and outcomes in upper gastrointestinal bleeding. Can J Gastroenterol 2009; 23: 489 493

[13] Jairath V, Kahan BC, Logan RF et al. Outcomes following acute nonvariceal upper gastrointestinal bleeding in relation to time to endoscopy: results from a nationwide study. Endoscopy 2012; 44: 723 - 730

[14] Greenspoon J, Barkun A, Bardou M et al. Management of patients with nonvariceal upper gastrointestinal bleeding. Clin Gastroenterol Hepatol 2012; 10: $234-239$

[15] Hwang JH, Fisher DA, Ben-Menachem T et al. The role of endoscopy in the management of acute non-variceal upper gastrointestinal bleeding. Gastrointest Endosc 2012; 75: $1132-1138$

[16] Laine L, Jensen DM. Management of patients with ulcer bleeding. Am J Gastroenterol 2012; 107: 345 - 360; quiz 361

[17] Abougergi MS, Travis AC, Saltzman JR. The in-hospital mortality rate for upper $\mathrm{Gl}$ hemorrhage has decreased over 2 decades in the United States: a nationwide analysis. Gastrointest Endosc 2015; 81: $882-$ 888.e881

[18] Hearnshaw SA, Logan RF, Lowe D et al. Use of endoscopy for management of acute upper gastrointestinal bleeding in the UK: results of a nationwide audit. Gut 2010; 59: 1022 - 1029

[19] Rosenstock SJ, Moller MH, Larsson $\mathrm{H}$ et al. Improving quality of care in peptic ulcer bleeding: nationwide cohort study of 13,498 consecutive patients in the Danish Clinical Register of Emergency Surgery. Am J Gastroenterol 2013; 108: 1449-1457

[20] Khamaysi I, Gralnek IM. Acute upper gastrointestinal bleeding (upper gastrointestinal bleeding) - initial evaluation and management. Best Pract Res Clin Gastroenterol 2013; 27: 633-638 
[21] Spiegel BM, Vakil NB, Ofman J]. Endoscopy for acute nonvariceal upper gastrointestinal tract hemorrhage: is sooner better? A systematic review Arch Intern Med 2001; 161: 1393 - 1404

[22] Tsoi KK, Ma TK, Sung JJ. Endoscopy for upper gastrointestinal bleeding: how urgent is it? Nat Rev Gastroenterol Hepatol 2009; 6: $463-$ 469

[23] Cipolletta L, Bianco MA, Rotondano R et al. Outpatient management for low-risk nonvariceal upper gastrointestinal bleeding: a randomized controlled trial. Gastrointest Endosc 2002; 55: 1-5
[24] Lai KC, Hui WM, Wong BC et al. A retrospective and prospective study on the safety of discharging selected patients with duodenal ulcer bleeding on the same day as endoscopy. Gastrointest Endosc 1997; 45: $26-30$

[25] Lee JG, Turnipseed S, Romano PS et al. Endoscopy-based triage significantly reduces hospitalization rates and costs of treating upper gastrointestinal bleeding: a randomized controlled trial. Gastrointest Endosc 1999; 50: $755-761$ 
- Appendix 1 ICD-9-CM Diagnostic and Procedure Codes.

\begin{tabular}{|c|c|}
\hline Description & ICD-9-CM Code \\
\hline \multicolumn{2}{|l|}{ UGIH } \\
\hline Bleeding esophageal varices & $456.0,456.2,456.20$ \\
\hline Bleeding peptic ulcer (gastric or duodenal) & $\begin{array}{l}\text { 531.0x, 531.2x, 531.4x, 531.6x, } \\
\text { 532.0x, 532.2x, 532.4x, 532.6x, } \\
\text { 533.0x, 533.2x, 533.4x, 533.6x, } \\
\text { 534.0x, 534.2x, 534.4x, 534.6x }\end{array}$ \\
\hline Mallory-Weiss & 530.7 \\
\hline Gastritis with hemorrhage & $535.01,535.11,535.21,535.41,535.51,535.61$ \\
\hline Dieulafoy's lesion of stomach or duodenum & 537.84 \\
\hline Angiodysplasia of stomach or duodenum with hemorrhage & 537.83 \\
\hline \multicolumn{2}{|l|}{ Other diagnoses } \\
\hline Acute renal failure (including dialysis) & $584.5,584.6,584.7,584.8,584.9, \mathrm{~V} 45.1, \mathrm{~V} 56.0, \mathrm{~V} 56.1$ \\
\hline Hypovolemia including shock & $276.5,276.50,276.51,276.52,785.59$ \\
\hline Acute respiratory failure & 518.81 \\
\hline \multicolumn{2}{|l|}{ Procedures } \\
\hline Blood transfusion & $99.03,99.04$ \\
\hline Dialysis (for acute renal failure definition) & 39.95 \\
\hline EGD & $45.13,45.14,45.16,42.23,44.13,42.33,44.43$ \\
\hline Endoscopic therapy at EGD & $42.33,44.43$ \\
\hline
\end{tabular}

